\title{
Validación del inventario multidimensional de acoso psicológico en universitarios
}

\author{
Validation of the Multidimensional Inventory of Psychological Abuse among \\ University Students
}

\author{
Juana Vianey Plata Santander* \\ Angélica Romero Palencia* \\ Arturo del Castillo Arreola* \\ Gloria Ángela Domínguez Aguirre** \\ Adriana Martínez Peralta** \\ Universidad Autónoma del Estado de Hidalgo* \\ Universidad Juárez Autónoma de Tabasco**
}

\section{RESUMEN}

Estadísticas relacionadas con el acoso escolar han producido resultados ambiguos en la prevalencia de esta problemática (Clémence, 2001; Namie, 2009). Una posible explicación a esto consiste en que algunas medidas carecen de validez y confiabilidad en la población estudiada. Así, la investigación tuvo como objetivo la validación en México de un Inventario Multidimensional de Acoso Psicológico en Universitarios (IMAPU). Participaron 489 estudiantes, 263 pertenecientes a la Universidad Autónoma del Estado de Hidalgo y 226 pertenecientes a la Universidad Juárez Autónoma de Tabasco. Se obtuvo una escala válida y confiable que consta de 34 ítems tipo Likert, con cinco opciones de respuesta, que muestra que el acoso en universitarios aplica tanto horizontalmente (entre pares) como verticalmente (de profesores hacia alumnos).

Palabras clave: agresión, medición, psicometría, universidad, violencia.

\section{ABSTRACT}

Statistics related to abuse at school have produced ambiguous results in determining the prevalence of this phenomenon (Clémence, 2001; Namie, 2009). A possible explanation for this could be that some measures lack validity and reliability among the population in question. Therefore, this research sought the validation in Mexico of a Multidimensional Inventory of Psychological Abuse among University Students. For this study, 489 students participated: 263 from the Autonomous University of the State of Hidalgo, and 226 from the "Juarez" Autonomous University of the State of Tabasco. A valid and reliable scale was obtained, consisting of 34 Likert-type items with five response options, which shows that abuse among university students occurs both horizontally (among peers) as well as vertically (teachers toward students).

Keywords: aggression, psychometry, university, violence. 
El acoso escolar ha sido investigado y medido en diversos estudios en subgrupos de diferentes edades y contextos (Adams \& Lawrence, 2011). En estos estudios se ha dejado fuera el ambiente universitario, a pesar de ser el periodo de transición entre la formación académica y la vida profesional.

Para Namie (2009), el acoso entre pares ha sido diferenciado de la violencia juvenil y se define como aquel que ocurre entre niños y adolescentes en el contexto escolar, que muestra una posición entre ellos generalmente precisada por los términos victimario y víctima. A partir de los estudios de Olweus (2006), este fenómeno ha sido objeto de una definición cada vez más consensuada, considerando al acoso ejercido sobre un compañero o compañera de aula como un conjunto de acciones tendientes a amedrentar, extorsionar, excluir y debilitar a la víctima en circunstancias grupales, esto es, con la presencia de los pares como testigos e incluye el daño físico.

$\mathrm{El}$ acoso que ocurre entre pares consiste en el establecimiento de una relación particular, producida y auspiciada socialmente por un régimen de inseguridad que opera sobre el miedo como vector de agenciamiento y tiende a instaurar la indefensión (Smith, 2011). Al ser un reflejo del funcionamiento social, es probable que el acoso se presente en otros ambientes educativos además de los infantiles, tal como el universitario.

Monks y Smith (2010) afirman que la agresión existe en todos los niveles de edad, en diversos grados, y explican formas de maltrato que identifican tipos de agresión presentes en el acoso escolar que podrían ocurrir en comunidades universitarias de la siguiente manera: 1) acoso de forma directa e indirecta, donde en un ataque directo la víctima identifica al agresor, en un ataque indirecto la víctima es dañada pero no sabe quién es el culpable; 2 ) acoso psicológico o verbal, una forma de agresión que por lo general se presenta en expresiones altamente agresivas o apodos despectivos que aluden defectos de las personas, y es más común que el maltrato físico, especialmente en jóvenes; 3) acoso relacional, que en muchos casos de maltrato es llamado maltrato relacional porque rompe las relaciones sociales entre las víctimas y sus pares. Aunque desde preescolar se puede presentar este tipo de agresión se hace más frecuente y dañina durante la pubertad, porque es cuando los adolescentes adquieren habilidades sociales y la aprobación de sus pares es importante; posteriormente en la adultez, la persona busca aprobación y gratificaciones que lo hagan sentirse aceptado y útil. Conforme mayor indiferencia y sentimiento de no aceptación en un grupo o una comunidad, mayor sentimiento de rechazo existirá en la persona.

Este tipo de conductas, al presentarse de manera constante, repetitiva, con intención de daño y con abuso de poder, ha constituido un fenómeno denominado bullying o acoso escolar. Este fenómeno se evidencia cada vez en más comunidades y a diferentes edades; incluso de acuerdo con Adams y Lawrence (2011) conforme más edad la causa de bullying sellena de matices y es más complejo detectarlo.

Por otra parte, Namie (2009) afirma que la intimidación o acoso psicológico puede considerarse como una incivilidad intensificada que tiene que ver con diversas esferas, tales como la familia, el contexto social y la cultura, además del contexto escolar. Este mismo autor recomienda no manejar la tendencia a explicar el bullying en función de factores de personalidad, sino considerando el contexto, es decir, los factores sociales y culturales en que se manifiesta.

La definición de acoso escolar se ha desarrollado durante los últimos 30 años en estudios que han variado sus medidas para incluir una amplia gama de conductas. Estas prácticas han llevado a diversas estimaciones de prevalencia: los índices de acoso escolar se han diferenciado dramáticamente a través de diferentes muestras, lo que ha dificultado la comparación y podría reflejar imprecisión de las medidas (Benites, 2012).

El acoso escolar puede tener diversos motivos desencadenantes, pero se compone de un hostigamiento constante y repetitivo. En consecuencia, a mayor edad, el papel del agresor va afianzando una conducta antisocial, cuyas consecuencias suelen provocar la exclusión social y la predelincuencia, o en un futuro llevar el fenómeno de acoso escolar hacia mobbing o acoso laboral (Ortega, 2010). Además, el clima afectivo del grupo sufre una importante pérdida de actitudes prosociales al favorecer la falta de consideración hacia los demás y la consiguiente merma en la calidad del clima educativo (Ortega, Del Rey \& Casas, 2013). Se trata, pues, de un fenómeno de amplia repercusión que 
afecta a toda la comunidad educativa y quebranta la propia sensación de seguridad.

Las consecuencias de este fenómeno social conducen a experimentar desprotección; en adolescentes y adultos jóvenes llevan al desamparo y en todas las edades pueden llegar a afecciones mentales y psicosomáticas; además, este fenómeno probablemente tenga consecuencias en la baja calidad de profesionistas en el país e incluso puede constituir un problema de salud pública (Romero-Palencia \& Plata, en prensa).

Sin embargo, no existe un instrumento de medición válido y confiable para la población mexicana que evalúe el acoso psicológico en universitarios y que permita identificar los elementos mencionados anteriormente.

Como marco de este estudio se contemplaron algunos instrumentos de medición con base en su utilidad y concordancia con los objetivos. Los instrumentos se eligieron según la probable identificación de conductas de agresión y hostigamiento que demuestren la existencia del bullying o acoso escolar; también se tomaron en cuenta instrumentos relacionados con investigaciones de mobbing ya que, como se mencionó antes, hay ciertos aspectos de convivencia en los universitarios que son semejantes a la que mantienen las personas en el medio laboral (Romero-Palencia \& Plata, en prensa).

\section{Cuestionario sobre intimidación y maltrato \\ entre iguales (CIMEI).}

El cuestionario conocido como CIMEI (Concepciones sobre Intimidación y Maltrato entre Iguales) (Avilés \& Monjas, 2005) es de tipo autoaplicable y está formado por tres secciones con 42 ítems en total, basados en otros cuestionarios sobre intimidación y maltrato entre iguales, de autores como Ortega, Mora y MoraMerchán (1995, en Avilés \& Monjas, 2005) y el cuestionario sobre abusos entre compañeros de Fernández García y Ortega (2001, en Avilés \& Monjas, 2005), los cuales pretenden indagar la relevancia para las situaciones de maltrato de los papeles que adoptan los diferentes participantes en él, en el seno del grupo de iguales.

\section{Cuestionario sobre convivencia, violencia y experiencias de riesgo (COVER).}

Es un instrumento compuesto de 54 ítems en una escala tipo Likert que aluden a situaciones ocurridas en los tres últimos meses. Fue elaborado en Nicaragua ad hoc, con el objetivo de establecer una línea base para el diseño de políticas preventivas ante la violencia escolar e identificar, de manera específica, el peso de las distintas formas de ejercer el dominio-sumisión: física, verbal, psicológica y social, donde básicamente se define el constructo bullying y se logra observar las posibles diferencias en función del género (Ortega \& Del Rey, 2003).

Cuestionario Individual sobre Psicoterror, Negación, Estigmatización y Rechazo en Organizaciones Sociales (Barómetro cisneros).

En general, la escala cisneros es un cuestionario desarrollado por Piñuel (2001, en Fidalgo \& Piñuel, 2004), de carácter autoadministrado y compuesto por 43 ítems tipo Likert en una escala de 0 (nunca) a 6 (todos los días), que objetivan y valoran 43 conductas de acoso psicológico, para sondear periódicamente el estado y las consecuencias de la violencia en el entorno laboral de las organizaciones.

Los instrumentos que se han encontrado en investigaciones son útiles para los objetivos, pero tienen como limitante el hecho de que fueron implementados en contextos diferentes al universitario. En adición, la carencia de estudios de acoso escolar interesados en contextos universitarios motivó la presente investigación, cuya finalidad consistió en crear y validar una escala para evaluar el acoso escolar en comunidades universitarias.

\section{MÉTODO}

\section{Participantes}

Se contó con 489 participantes voluntarios en educación universitaria, 141 hombres (29\%) y 348 mujeres (71\%), con un promedio de edad de 20 años y un rango de 18-34 años de edad; 263 alumnos (54\%) pertenecen a la Universidad Autónoma del Estado de Hidalgo (UAEH) matriculados en el Instituto de Ciencias de la Salud (Icsa) y 226 alumnos (46\%) de la Universidad Juárez Autónoma de Tabasco (UJAT), de los cuales 314 alumnos (64\%) eran de la licenciatura en psicología, 49 (10\%) de la licenciatura en medicina, 35 (7\%) de la licenciatura en enfermería, 30 (6\%) de la licenciatura en gerontología, $26(5 \%)$ de la licenciatura en nutrición, $23(5 \%)$ de la licenciatura en odontología y 12 (3\%) de la licenciatura en farmacia. 


\section{Instrumento}

Con base en los resultados del análisis de contenido del estudio exploratorio se tomaron los indicadores más frecuentemente utilizados por categoría, y se enmarcaron en afirmaciones con forma de respuesta tipo Likert de seis puntos (Romero-Palencia y Plata, en prensa).

En su primera versión, la subescala de convivencia entre alumnos y maestros constó de 39 reactivos en intervalos de tiempo ("nunca", "menos de una vez al semestre", "menos de una vez al mes", "algunas veces al mes", "una vez a la semana", "varias veces a la semana", "todos los días"). La subescala de convivencia entre pares constó de 39 reactivos también en intervalos de tiempo ("nunca", "menos de una vez al semestre", "menos de una vez al mes", "algunas veces al mes", "una vez a la semana", "varias veces a la semana", "todos los días"). Finalmente, la subescala de víctimización estuvo formada por 18 reactivos igualmente en intervalos de tiempo ("nunca", "menos de una vez al semestre", "menos de una vez al mes", "algunas veces al mes", "una vez a la semana", "varias veces a la semana", "todos los días").

\section{Procedimiento}

Los ítems de la primera versión se sometieron a una validación por jueces y arrojaron 90\% de acuerdo. Después de realizar el piloteo y los ajustes de lenguaje necesarios se procedió a la aplicación de la primera versión de la Escala de Acoso Psicológico en Universitarios (IMAPU).

Se les pidió a los participantes que respondieran a la escala de la manera más clara y sincera posible, y se les indicó que el cuestionario formaba parte de una investigación, cuyo objetivo era conocer la existencia del acoso escolar en comunidades universitarias. Asimismo, se destacó el anonimato de las respuestas y se señaló que las respuestas no serían catalogadas como buenas o malas, correctas o incorrectas, con el fin de garantizar la confidencialidad de los participantes.

\section{RESULTADOS}

Una vez terminada la aplicación de los instrumentos se procedió al análisis psicométrico de la escala, el cual se dividió en dos partes.

Se decidió eliminar aquellos reactivos que no cumplieron con dos de los tres criterios requeridos para la discriminación del inventario: casos en todas las opciones de respuesta, correlación del reactivo con la escala total, diferencias significativas entre grupos extremos obtenidas mediante la prueba t de Student. Para la subescala de convivencia entre maestros y alumnos, del total de 39 reactivos nueve no cumplieron con estos requisitos. Asimismo, en la subescala de convivencia entre pares (entre alumnos) del total de 39 reactivos nueve no cumplieron con dos de los criterios.

Posteriormente, se realizó un análisis factorial de los componentes principales con rotación Varimax, por cada subescala, para obtener la validez del constructo; las propiedades psicométricas de validez y confiabilidad de cada subescala se presentan a continuación.

\section{Subescala de convivencia entre alumnos y maestros}

Se aplicó un análisis factorial de componentes principales, con rotación de normalización Varimax del cual se eligieron cinco factores con un valor propio mayor a 1, que explicaban $67.38 \%$ de la varianza. Asimismo, se eligieron sólo aquellos reactivos que tenían un peso factorial mayor o igual a .30 para conformar el instrumento final. La versión final quedó constituida con 30 reactivos ( vid. tabla 1). Posteriormente, se realizó la prueba del Alfa de Cronbach con el fin de conocer la consistencia interna de la subescala en su totalidad, resultando un Alfa total de .885. También se calculó la consistencia interna de cada factor.

\section{Subescala de convivencia entre pares}

Se aplicó un análisis factorial de componentes principales, con rotación de Normalización Varimax, del cual se eligieron los cinco factores con un valor propio mayor a 1 , que explicaban $64.74 \%$ de la varianza. Se eligieron sólo aquellos reactivos que tenían un peso factorial mayor o igual de .40 para confirmar el instrumento final. La versión final quedó constituida con 30 reactivos ( $v i d$. tabla 2). La consistencia interna de la subescala total fue de .911. También se calculó la consistencia interna de cada factor. 
Tabla 1. Análisis factorial de los reactivos que componen la subescala de convivencia entre maestros y alumnos

Reactivos

Factores

Factor 1. Intencionalidad del daño

Me han aturdido o gritado, o han elevado la voz de manera intimidante

Han inventado y difundido rumores y calumnias acerca de mí de manera malintencionada

Me han jalado, empujado o retado físicamente para intimidarme

Han hecho burla de mí o bromas intentando ridiculizar mi forma de hablar, de andar, de comportarme, etc.

Han controlado aspectos de mis actividades escolares de forma malintencionada para intentar "ponerme un cuatro"

Me han humillado, minusvalorado y echado por tierra mi trabajo, no importa lo que haya hecho

He recibido feroces e injustas críticas acerca de aspectos de mi vida personal

Han intentado provocarme o fastidiarme para "hacerme explotar"

\begin{tabular}{c|c|c|c|c} 
I & II & III & IV & V \\
.797 & & & & \\
\hline .791 & & & & \\
.774 & & & & \\
.749 & & & & \\
\hline .680 & & & & \\
\hline .580 & & & & \\
\hline .576 & & & & \\
\hline .497 & & & & \\
\hline
\end{tabular}

\section{Factor 2. Agresión directa}

Me han molestado o agredido en eventos fuera de la escuela como eventos académicos y actividades extracurriculares

Me han lanzado insinuaciones o proposiciones sexuales directas o indirectas

Me han considerado "inferior"

Me han hecho bromas pesadas cuando no "me llevo"

Se han burlado de mí, me han criticado o ridiculizado mis participaciones en clase

He recibido amenazas verbales o mediante gestos intimidatorios

Han utilizado varias formas de hacerme incurrir en errores sobre mis actividades escolares de manera malintencionada

Han intentado persistentemente desmoralizarme

\begin{tabular}{l|l|l|l|l} 
& & & & \\
\hline & .878 & & & \\
\hline & .772 & & & \\
\hline & .725 & & & \\
\hline & .664 & & & \\
\hline & .544 & & & \\
\hline & .523 & & & \\
\hline & .475 & & & \\
\hline
\end{tabular}

Factor 3. Abuso de autoridad

Han distorsionado malintencionadamente lo que digo o hago en mis actividades escolares

He recibido críticas y reproches por cualquier cosa que haga o decisión que tome en mis actividades escolares

Se han amplificado y dramatizado de manera injustificada errores pequeños o intrascendentes

Me han amenazado con usar instrumentos disciplinarios

\begin{tabular}{l|l|l|l|l} 
& & & & \\
\hline & & .762 & & \\
\hline & & .725 & & \\
\hline & & .714 & & \\
\hline
\end{tabular}


Factor 4. Carga injustificada de actividades escolares

Me han menospreciado personal o profesionalmente

Han intentado aislarme de mis compañeros dándome trabajos o tareas que me han alejado físicamente de ellos

Me han abrumado con una carga de trabajo insoportable de manera malintencionada

Me han ocasionado gastos con intención de perjudicarme económicamente

Me han asignado tareas o trabajos absurdos o sin sentido

Me han asignado tareas que ponen en peligro mi integridad física o mi salud a propósito

\begin{tabular}{c|c|c|c|c} 
I & II & III & IV & V \\
\hline & & & .827 & \\
\hline & & & .788 & \\
\hline & & & .701 & \\
\hline & & & .350 & \\
\hline
\end{tabular}

\section{Factor 5. Agresión indirecta}

Han modificado mis responsabilidades o las tareas a realizar sin decirme nada

Han minimizado continuamente mi esfuerzo profesional y académico

Me han asignado plazos o cargas de actividades escolares irracionales

Me han privado de información imprescindible y necesaria para hacer mis actividades escolares

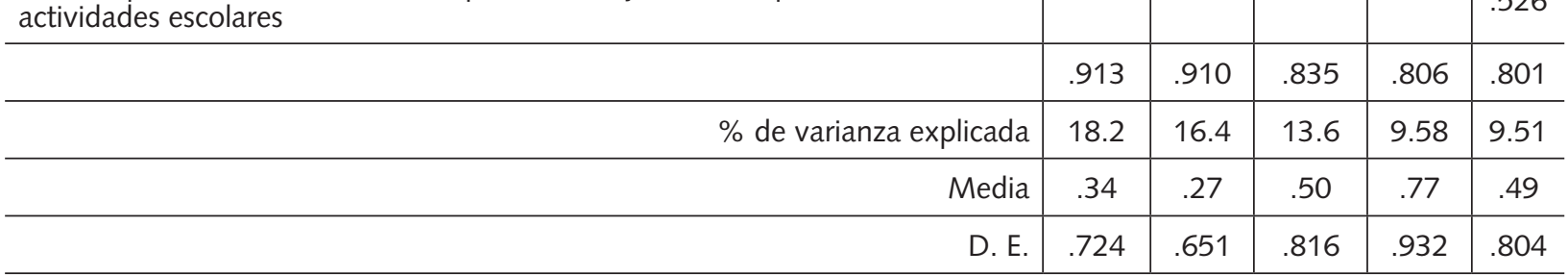

Tabla 2. Análisis factorial de los reactivos que componen la subescala de convivencia entre pares

\section{Reactivos}

\section{Factores}

\begin{tabular}{|c|c|c|c|c|c|}
\hline & I & II & III & IV & V \\
\hline Me han golpeado para intimidarme & .806 & & & & \\
\hline Me han acorralado u obstruido el paso para fastidiarme & .714 & & & & \\
\hline Se han burlado de mí, me han criticado o ridiculizado mis participaciones en clase & .707 & & & & \\
\hline Han hecho bromas inapropiadas y crueles acerca de mí & .698 & & & & \\
\hline Me han jalado, empujado o retado físicamente para intimidarme & .693 & & & & \\
\hline $\begin{array}{l}\text { He recibido amenazas por escrito, por teléfono, en mi domicilio, o a través de } \\
\text { medios electrónicos y redes sociales }\end{array}$ & .685 & & & & \\
\hline Me han gritado o elevado la voz de manera intimidante & .668 & & & & \\
\hline Me han puesto apodos o sobrenombres que me molestan & .653 & & & & \\
\hline Me han insultado o agredido verbalmente & .588 & & & & \\
\hline
\end{tabular}




\begin{tabular}{|c|c|c|c|c|c|}
\hline & I & II & III & IV & V \\
\hline He recibido amenazas verbales o mediante gestos intimidatorios & .578 & & & & \\
\hline Me han molestado o agredido en eventos fuera de la escuela como fiestas & .493 & & & & \\
\hline $\begin{array}{l}\text { Cuando se ha hecho trabajo en equipo han modificado mis responsabilidades } \\
\text { o las tareas a ejecutar sin decirme nada }\end{array}$ & & .822 & & & \\
\hline Han intentado persistentemente desmoralizarme & & .736 & & & \\
\hline $\begin{array}{l}\text { Han controlado aspectos de mis actividades escolares de forma } \\
\text { malintencionada para intentar "ponerme un cuatro" }\end{array}$ & & .724 & & & \\
\hline $\begin{array}{l}\text { Cuando se ha hecho trabajo en equipo he recibido una presión indebida para } \\
\text { sacar adelante las actividades escolares }\end{array}$ & & .681 & & & \\
\hline Me han ocasionado gastos con intención de perjudicarme económicamente & & .599 & & & \\
\hline $\begin{array}{l}\text { Cuando se ha hecho trabajo en equipo me han asignado tareas o trabajos } \\
\text { absurdos o sin sentido }\end{array}$ & & .578 & & & \\
\hline Han intentado provocarme o fastidiarme para "hacerme explotar" & & .570 & & & \\
\hline Me han minusvalorado continuamente mi esfuerzo académico y profesional & & .474 & & & \\
\hline Me han humillado, despreciado o minusvalorado en público & & & .698 & & \\
\hline Me han menospreciado personal o académicamente & & & .664 & & \\
\hline $\begin{array}{l}\text { Han hecho burla de mí o bromas intentando ridiculizar mi forma de hablar, de } \\
\text { andar, de comportarme, etc. }\end{array}$ & & & .620 & & \\
\hline $\begin{array}{l}\text { Me han lanzado insinuaciones o proposiciones sexuales desagradables directas } \\
\text { o indirectas }\end{array}$ & & & .574 & & \\
\hline Me han consideran "inferior" & & & .476 & & \\
\hline Me han robado pertenencias para fregarme & & & & .695 & \\
\hline $\begin{array}{l}\text { Han inventado y difundido rumores y calumnias acerca de mí de manera } \\
\text { malintencionada }\end{array}$ & & & & .646 & \\
\hline $\begin{array}{l}\text { Me han atribuido malintencionadamente conductas ilícitas o antiéticas para } \\
\text { perjudicar mi imagen y reputación }\end{array}$ & & & & .567 & \\
\hline Me han acusado injustificadamente de incumplimientos, errores o fallos & & & & & .810 \\
\hline $\begin{array}{l}\text { Me han minusvalorado y echado por tierra mi trabajo, no importa lo que } \\
\text { haya hecho }\end{array}$ & & & & & .788 \\
\hline \multirow[t]{2}{*}{$\begin{array}{l}\text { He recibido críticas y reproches por cualquier cosa que haga o decisión que } \\
\text { tome en mis actividades escolares }\end{array}$} & & & & & .670 \\
\hline & 933 & .901 & .842 & .801 & .865 \\
\hline$\%$ de varianza explicada & 20.4 & 14.2 & 11.4 & 9.4 & 9.1 \\
\hline Media & .231 & .384 & .372 & .215 & .458 \\
\hline D. E. & .536 & .689 & .673 & .602 & .812 \\
\hline
\end{tabular}




\section{DISCUSIÓN}

A partir de los resultados obtenidos en este estudio se obtuvo una escala válida y confiable para evaluar el acoso escolar en ambientes universitarios.

Sandoval et al. (2011) afirman que el fenómeno del acoso escolar (bullying) tiende a estar conformado por tres partes: la intención, la repetición o temporalidad y el desequilibrio de comunicación o abuso de poder. Estas áreas son evaluadas por la presente escala. En este caso, la agresión está enfocada a la competencia y a la necesidad de supremacía en relación con el aprendizaje y el conocimiento, desarrollada en dos tipos de convivencia: la primera es la que se genera entre pares, asociada a los intereses personales, tiene también las características de ser constante y repetitiva a través del tiempo y con la intención expresa de menoscabar la integridad del agredido. En este sentido, Yescas-Sánchez (2013) considera que el bullying consiste en aquellas agresiones que son actitudes o situaciones graves, dañinas, perjudiciales, que pueden tener una incidencia y permanencia por un tiempo suficiente para generar daño; pueden ser morales, sociales, psicológicas y físicas entre víctima y agresor, sin importar el sexo ni la edad escolar.

Es necesario realizar un análisis psicosocial del propio sistema de normas, valores, sentimientos y comportamientos de todas las personas que están detrás de estas acciones de maltrato y de violencia. Olweus (2006) considera que la agresión siempre está acompañada por una intencionalidad, esto como punto importante para diferenciar la verdadera agresión de aquellos comportamientos que, de forma involuntaria, provocan algún tipo de efecto perjudicial en los demás.

La convivencia entre maestros y alumnos está asociada a la incapacidad y descontrol de enseñanza en los grupos; según Olweus (2006) esto es el desequilibrio de poder entre la víctima y su agresor. Este factor se traduce en el deseo de control por parte del agresor sobre lo que ocurre en la víctima y lo que siente. Este dominio necesario de la situación se acompaña por la indefensión que va creciendo en las víctimas a medida que van ocurriendo los episodios violentos. La combinación de ambos aspectos genera una dinámica de dominiosumisión que no necesita la presencia física del agresor, sino que permanece bajo la influencia de éste en cualquier momento.

Dentro de los objetivos del acoso escolar en los universitarios están las perturbaciones a la integridad a través de un abuso de autoridad o de poder, burlas como bromas y apodos. Así, este tipo de acoso escolar también considera algunas semejanzas con el acoso laboral, ya que este tipo se caracteriza por el ataque, de manera ilegal y tumultuosa, de parte de un grupo de personas hacia otro completamente indefenso e intimidado por la mayoría. Otras de las semejanzas que tiene el acoso laboral con el que se estudia en la presente investigación es que se acompaña de una comunicación hostil con falta de ética que empuja a la víctima a una actitud de desesperanza e indefensión (Yescas-Sánchez, 2013). De esta forma, parecería que el acoso escolar en universidades es una violencia con algunas características del acoso escolar tradicional, y otras del acoso laboral. El acoso escolar tradicional se presenta como agresividad intencional, y el laboral, como agresividad por supremacía.

Finalmente, en cuanto a los involucrados, el bullying tradicional sólo considera a los infantes pares o iguales, y el mobbing toma en cuenta el comportamiento del adulto entre subordinados y jefes/superiores; en el caso del acoso en universitarios, se consideran adultos jovenes involucrados en una agresión sutil y emascarada, ya sea entre pares o de un superior a un subordinado (maestro-alumno). Pareciera que la dinámica del bullying va transformándose a lo largo del desarrollo de los individuos, refinándose, dirigiéndose a agresiones sutiles que se prueban en el medio universitario y que se asemejan al acoso laboral. 


\section{REFERENCIAS}

Adams, F.D. \& Lawrence, G. J. (2011). "Bullying victims: The effects last into college". En: Informe sobre la Convivencia en los Centros Educativos. España.

Avilés, J. M. \& Monjas, C. I. (2005). "Estudio de la incidencia de la intimidación y el maltrato entre iguales en la educación secundaria obligatoria mediante el cuestionario cimei". (Cuestionario sobre Intimidación y Maltrato entre Iguales). Anales de Psicología, 21(1), 27-41.

Benites, M. (2012). "La convivencia escolar: una estrategia de intervención en bullying”. Revista Cultura, en prensa. Recuperado de: http://alfepsi.org/attachments/articleLaconvivenciaescolarunaestrategiadeintervencenbullyingLuisBenites.pdf

Fidalgo, A. \& Piñuel, I. (2004). La escala de Cisneros como herramienta de valoración del Mobbing. Revista Psicothema, 16(4), 615-624. Recuperado de: http://www.psicothema.com

Monks, C. P. \& Smith, P. K. (2010). Definitions of bullying: Age differences in understanding of the term and the role of experience. British Journal of Developmental Psychology, 24(4), 801-821.

Namie G. (2009). Bullying y contexto ambiental. Recuperado de: http://www.colegiointernacional. edu .mx|tips-bullying-com.pdf

Observatorio sobre Violencia y Convivencia en la Escuela. (2011). Impacto social de la violencia escolar. Informe preliminar. Lima:

Olweus, D. (2006). Bullying: problema adolescente. Entrevista con J. M. Lacasa: La mirada de Jokin. Recuperado de: www.argijokin.blogeindario. com|2006|01|04927-dan-olweus
Ortega, R. \& Del Rey. R. (2003). Cuestionario sobre convivencia, violencia y experiencias de riesgo - cover. Documento no publicado.

Ortega-Ruiz, R., Del Rey, R. \& Casas, J.A. (2013). La convivencia escolar: clave en la predicción del Bullying. Revista Iberoamericana de Evaluación Educativa, 6(2), 91-102

Ortega, R. (2010). Agresividad injustificada, bullying y violencia escolar. Madrid: Alianza Editorial.

Prieto, Q. \& Carrillo, N. (2009). Fracaso escolar y su vínculo con el maltrato entre alumnos: el aula como escenario de la vida afectiva. Revista Iberoamericana de Educación, 49(5), 1-8.

Salmivalli, C. (2010). Bullying and the peer group: A review. Aggression and violent behavior, 15(2), 112-120.

Sandoval, E., González, C., Méndez, P., Pérez, G., Aguirre, C., Nájera, V., Hernández, M., Téllez, M., Chinchilla, M., González, R. \& Gómez, R. (2011). El bullying como un fenómeno psicosocial silencioso en las instituciones educativas oficiales de educación básica del municipio de Jalapa. México: Universidad Panamericana, Facultad de Ciencias de la Educación, Profesorado en Psicología.

Smith, K. (2011). Anoka-Hennepin sued over bullying. Minneapolis: Star Tribune. July. Recuperado de: http://www.startribune.com/local/north/125958688. html.

Yescas-Sánchez, R. (2013). Intervención educativa para resolver un caso de acoso escolar. Revista Semestral da Associação Brasileira de Psicologia Escolar e Educacional, 17(2), 339-354. 